\title{
NON-ARCHIMEDEAN GENERALIZED BESSEL POTENTIALS AND THEIR APPLICATIONS
}

\author{
ANSELMO TORRESBLANCA-BADILLO
}

\begin{abstract}
This article describes a class of pseudo-differential operators

$$
\left(\mathcal{A}^{\alpha} \varphi\right)(x)=\mathcal{F}_{\xi \rightarrow x}^{-1}\left(\left[\max \left\{\left|\boldsymbol{\psi}_{1}\left(\|\xi\|_{p}\right)\right|,\left|\boldsymbol{\psi}_{2}\left(\|\xi\|_{p}\right)\right|\right\}\right]^{-\alpha} \widehat{\varphi}(\xi)\right),
$$

$\varphi \in \mathcal{D}\left(\mathbb{Q}_{p}^{n}\right)$ and $\alpha \in \mathbb{C}$; here $\left[\max \left\{\left|\boldsymbol{\psi}_{1}\left(\|\xi\|_{p}\right)\right|,\left|\boldsymbol{\psi}_{2}\left(\|\xi\|_{p}\right)\right|\right\}\right]^{-\alpha}$ is the symbol of the operator $\mathcal{A}^{\alpha}$. These operators can be seen as a generalization of the Bessel potentials in the $p$-adic context. We show that the family $\left(K_{\alpha}\right)_{\alpha>0}$ of convolution kernels attached to generalized Bessel potentials $\mathcal{A}^{\alpha}, \alpha>0$, determine a convolution semigroup on $\mathbb{Q}_{p}^{n}$. Imposing certain conditions we have that $K_{\alpha}, \alpha>0$, is a probability measure on $\mathbb{Q}_{p}^{n}$. Moreover, we will study certain properties corresponding to the Green function of the operator $\mathcal{A}^{\alpha}$ and we show that heat equations, naturally associated to these operators, describes the cooling (or loss of heat) in a given region over time.
\end{abstract}

\section{INTRODUCTION}

In the latest years, there has been a strong interest on the non-archimedean pseudo-differential operators due its connections with $p$-adic pseudo-differential equations that describe certain physical models, see e.g. [1, [2], [3, 6], [7, 10], [13], 14, 15, [19, [21, and the references therein. Particularly, pseudo-differential operators whose symbols are associated with negative definite functions on the $p$ adic numbers, see [8, 9], [17, [18].

In this article, we introduce a new class of non-archimedean pseudo-differential operators (called the generalized Bessel potentials) associated with negative definite functions in the $p$-adic context and in arbitrary dimension. These operators have the form

$$
\left(\mathcal{A}^{\alpha} \varphi\right)(x)=\mathcal{F}_{\xi \rightarrow x}^{-1}\left(\left[\max \left\{\left|\boldsymbol{\psi}_{1}\left(\|\xi\|_{p}\right)\right|,\left|\boldsymbol{\psi}_{2}\left(\|\xi\|_{p}\right)\right|\right\}\right]^{-\alpha} \widehat{\varphi}(\xi)\right), \quad \varphi \in \mathcal{D}\left(\mathbb{Q}_{p}^{n}\right),
$$

where $\alpha \in \mathbb{C}, \mathcal{F}_{\xi \rightarrow x}^{-1}$ denotes the inverse Fourier transform, $\widehat{\varphi}$ is the Fourier transform of $\varphi, \mathcal{D}\left(\mathbb{Q}_{p}^{n}\right)$ denotes the $\mathbb{C}$ - vector space of Bruhat-Schwartz functions over $\mathbb{Q}_{p}^{n}$ and the function $\left[\max \left\{\left|\boldsymbol{\psi}_{1}\left(|| \xi \|_{p}\right)\right|,\left|\boldsymbol{\psi}_{2}\left(\| \xi||_{p}\right)\right|\right\}\right]^{-\alpha}$ is called symbol of the operator $\mathcal{A}^{\alpha}$. The functions $\boldsymbol{\psi}_{1}, \boldsymbol{\psi}_{2}: \mathbb{Q}_{p}^{n} \rightarrow \mathbb{C}$ are continuous, negative definite and radials. Taking, $\boldsymbol{\psi}_{1}=1$ and $\boldsymbol{\psi}_{2}=\|\cdot\|_{p}$, then by [4, Proposition 7.4-(iii)] and [17, Example 3.4] we have that $\mathcal{A}^{\alpha}$ correspond to the Bessel potential studied at [9] and [16].

We are interested in the pseudo-differential operators $\mathcal{A}^{\alpha}$ due to our interest for

Key words and phrases. Pseudo-differential operators, convolution kernels, convolution semigroups, Green's function, heat kernel, $p$-adic numbers, non-archimedean analysis.

* Anselmo Torresblanca-Badillo atorresblanca@uninorte.edu.co. 
contemporary physical theories, in particular, study new Cauchy problems (or $p$ adic heat equations), which governs the temperature distribution in an object over time. The corresponding equations takes the form

$$
\left\{\begin{array}{l}
\frac{\partial u}{\partial t}(x, t)=\mathcal{A}^{\alpha} u(x, t), \quad t \in[0, \infty), \quad x \in \mathbb{Q}_{p}^{n} \\
u(x, 0)=u_{0}(x) \in \mathcal{D}\left(\mathbb{Q}_{p}^{n}\right) .
\end{array}\right.
$$

It is worth noting that is that unlike the fundamental solutions studied at $[3,[8$, [1], [13, [17, 18, [21, et al., in our case, the fundamental solutions $Z(x, t)$ of the new Cauchy problems satisfies $Z(x, t) \leq 0$ for all $x \in \mathbb{Q}_{p}^{n} \backslash\{0\}$ and $t>0$, see Theorem 4. Therefore, we have observed that the new above $p$-adic heat equations, give temperatures less than absolute zero, which means from the physical point of view, that this equations describe the loss of heat over time.

There are many physical and mathematical motivations to employ $p$-adic analysis in investigation of mathematical and theoretical aspects of modern quantum physics, such as the properties of the Green's function over the field of $p$-adic numbers. Green's functions are a necessary stage in the formulation of the new $p$-adic quantum theory. For further details the reader may consult [5, 12], 20, and the references therein.

Motivated by the above, in this article we introduce a new class of $n$-dimensional $p$-adic Green function $G$ attached to pseudo-differential operator $\mathcal{A}^{\alpha}$ and given by

$$
G(x):=\mathcal{F}_{\xi \rightarrow x}^{-1}\left(\frac{1}{m^{2}+\left[\max \left\{\left|\psi_{1}\left(|| \xi \|_{p}\right)\right|,\left|\psi_{2}\left(\|\xi\|_{p}\right)\right|\right\}\right]^{-\alpha}}\right), m>0 .
$$

On the other hand, since the analysis of convolution semigroups of probability measures on locally compact abelian groups play an important role in probability theory, functional analysis and potential theory, see e.g. [4. In this article, we are interested in obtaining a new family of convolution semigroups of probability measures on $\mathbb{Q}_{p}^{n}$.

The article is organized as follows: In Section 2, we will collect some basic results on the $p$-adic analysis and fix the notation that we will use through the article. In Section 3, we will introduce a new class of non-archimedean pseudo-differential operators on the space $\mathcal{D}\left(\mathbb{Q}_{p}^{n}\right)$ which we call generalized Bessel potentials. In Section 4, we study certain properties corresponding to the convolution kernels $K_{\alpha}$, attached to generalized Bessel potentials, see Theorem 1 and Corollary 1 . Moreover, we show that the family $\left(K_{\alpha}\right)_{\alpha>0}$ determine a convolution semigroup on $\mathbb{Q}_{p}^{n}$, see Theorem 2. Imposing certain conditions we have that $K_{\alpha}, \alpha>0$, is a probability measure on $\mathbb{Q}_{p}^{n}$, see Corollary 2. In Section 5, we will study certain properties corresponding to the Green function and the heat Kernel attached to operator $\mathcal{A}^{\alpha}$, see Theorem 3 and Theorem 4 , respectively.

\section{Fourier Analysis on $\mathbb{Q}_{p}^{n}$ : Essential Ideas}

2.1. The field of $p$-adic numbers. Along this article $p$ will denote a prime number. The field of $p$-adic numbers $\mathbb{Q}_{p}$ is defined as the completion of the field of rational numbers $\mathbb{Q}$ with respect to the $p$-adic norm $|\cdot|_{p}$, which is defined as

$$
|x|_{p}= \begin{cases}0, & \text { if } \quad x=0 \\ p^{-\gamma}, & \text { if } \quad x=p^{\gamma} \frac{a}{b},\end{cases}
$$


where $a$ and $b$ are integers coprime with $p$. The integer $\gamma:=\operatorname{ord}(x)$, with $\operatorname{ord}(0):=$ $+\infty$, is called the $p$-adic order of $x$.

Any $p$-adic number $x \neq 0$ has a unique expansion of the form

$$
x=p^{\operatorname{ord}(x)} \sum_{j=0}^{\infty} x_{j} p^{j},
$$

where $x_{j} \in\{0,1,2, \ldots, p-1\}$ and $x_{0} \neq 0$. By using this expansion, we define the fractional part of $x \in \mathbb{Q}_{p}$, denoted $\{x\}_{p}$, as the rational number

$$
\{x\}_{p}= \begin{cases}0, & \text { if } \quad x=0 \text { or } \operatorname{ord}(x) \geq 0 \\ p^{\operatorname{ord}(x)} \sum_{j=0}^{-\operatorname{ord} d_{p}(x)-1} x_{j} p^{j}, & \text { if } \quad \operatorname{ord}(x)<0 .\end{cases}
$$

We extend the $p$-adic norm to $\mathbb{Q}_{p}^{n}$ by taking

$$
\|x\|_{p}:=\max _{1 \leq i \leq n}\left|x_{i}\right|_{p}, \text { for } x=\left(x_{1}, \ldots, x_{n}\right) \in \mathbb{Q}_{p}^{n} .
$$

For $r \in \mathbb{Z}$, denote by $B_{r}^{n}(a)=\left\{x \in \mathbb{Q}_{p}^{n} ;\|x-a\|_{p} \leq p^{r}\right\}$ the ball of radius $p^{r}$ with center at $a=\left(a_{1}, \ldots, a_{n}\right) \in \mathbb{Q}_{p}^{n}$, and take $B_{r}^{n}(0)=: B_{r}^{n}$. Note that $B_{r}^{n}(a)=$ $B_{r}\left(a_{1}\right) \times \cdots \times B_{r}\left(a_{n}\right)$, where $B_{r}\left(a_{i}\right):=\left\{x \in \mathbb{Q}_{p} ;\left|x_{i}-a_{i}\right|_{p} \leq p^{r}\right\}$ is the onedimensional ball of radius $p^{r}$ with center at $a_{i} \in \mathbb{Q}_{p}$. The ball $B_{0}^{n}$ equals the product of $n$ copies of $B_{0}=\mathbb{Z}_{p}$, the ring of $p$-adic integers of $\mathbb{Q}_{p}$. We also denote by $S_{r}^{n}(a)=\left\{x \in \mathbb{Q}_{p}^{n} ;\|x-a\|_{p}=p^{r}\right\}$ the sphere of radius $p^{r}$ with center at $a=\left(a_{1}, \ldots, a_{n}\right) \in \mathbb{Q}_{p}^{n}$, and take $S_{r}^{n}(0)=: S_{r}^{n}$. The balls and spheres are both open and closed subsets in $\mathbb{Q}_{p}^{n}$.

As a topological space $\left(\mathbb{Q}_{p}^{n},\|\cdot\|_{p}\right)$ is totally disconnected, i.e. the only connected subsets of $\mathbb{Q}_{p}^{n}$ are the empty set and the points. A subset of $\mathbb{Q}_{p}^{n}$ is compact if and only if it is closed and bounded in $\mathbb{Q}_{p}^{n}$, see e.g. [19, Section 1.3], or [2, Section 1.8]. The balls and spheres are compact subsets. Thus $\left(\mathbb{Q}_{p}^{n},\|\cdot\|_{p}\right)$ is a locally compact topological space.

We will use $\Omega\left(p^{-r}\|x-a\|_{p}\right)$ to denote the characteristic function of the ball $B_{r}^{n}(a)$. We will use the notation $1_{A}$ for the characteristic function of a set $A \subset \mathbb{Q}_{p}^{n}$. Along the article $d^{n} x$ will denote a Haar measure on $\mathbb{Q}_{p}^{n}$ normalized such that $\int_{\mathbb{Z}_{p}^{n}} d^{n} x=1$.

2.2. Some function spaces. A complex-valued function $f$ defined on $\mathbb{Q}_{p}^{n}$ is called locally constant if for any $x \in \mathbb{Q}_{p}^{n}$ there exist an integer $l:=l(x)$ such that

$$
f(x)=f\left(x^{\prime}\right) \text { for all } x^{\prime} \in B_{l}^{n}(x) .
$$

Equivalently, there exists a clopen partition $U$ of $\mathbb{Q}_{p}^{n}$ such that $f$ is constant on each element of $U$.

Denote by $\varepsilon\left(\mathbb{Q}_{p}^{n}\right)$ the linear space of locally constant $\mathbb{C}$-value functions on $\mathbb{Q}_{p}^{n}$.

A function $\varphi: \mathbb{Q}_{p}^{n} \rightarrow \mathbb{C}$ is called a Bruhat-Schwartz function (or a test function) if it is locally constant with compact support.

The $\mathbb{C}$-vector space of Bruhat-Schwartz functions is denoted by $\mathcal{D}\left(\mathbb{Q}_{p}^{n}\right)=: \mathcal{D}$. Let $\mathcal{D}^{\prime}\left(\mathbb{Q}_{p}^{n}\right)=: \mathcal{D}^{\prime}$ denote the set of all continuous functional (distributions) on $\mathcal{D}$. The natural pairing $\mathcal{D}^{\prime}\left(\mathbb{Q}_{p}^{n}\right) \times \mathcal{D}\left(\mathbb{Q}_{p}^{n}\right) \rightarrow \mathbb{C}$ is denoted as $\langle T, \varphi\rangle$ for $T \in \mathcal{D}^{\prime}\left(\mathbb{Q}_{p}^{n}\right)$ and $\varphi \in \mathcal{D}\left(\mathbb{Q}_{p}^{n}\right)$, see e.g. [2, Section 4.4].

Denote by $L_{\text {loc }}^{1}\left(\mathbb{Q}_{p}^{n}\right)$ the set of functions $f: \mathbb{Q}_{p}^{n} \rightarrow \mathbb{C}$ such that $f \in L^{1}(K)$ for any 
compact $K \subset \mathbb{Q}_{p}^{n}$. Every $f \in L_{l o c}^{1}\left(\mathbb{Q}_{p}^{n}\right)$ defines a distribution $f \in \mathcal{D}^{\prime}\left(\mathbb{Q}_{p}^{n}\right)$ by the formula

$$
\langle f, \varphi\rangle=\int_{\mathbb{Q}_{p}^{n}} f(x) \varphi(x\rangle d^{n} x .
$$

Such distributions are called regular distributions.

Given $\rho \in[0, \infty)$, we denote by $L^{\rho}\left(\mathbb{Q}_{p}^{n}, d^{n} x\right)=L^{\rho}\left(\mathbb{Q}_{p}^{n}\right):=L^{\rho}$, the $\mathbb{C}$-vector space of all the complex valued functions $g$ satisfying $\int_{\mathbb{Q}_{p}^{n}}|g(x)|^{\rho} d^{n} x<\infty, L^{\infty}$ $:=L^{\infty}\left(\mathbb{Q}_{p}^{n}\right)=L^{\infty}\left(\mathbb{Q}_{p}^{n}, d^{n} x\right)$ denotes the $\mathbb{C}$-vector space of all the complex valued functions $g$ such that the essential supremum of $|g|$ is bounded.

Let denote by $C\left(\mathbb{Q}_{p}^{n}, \mathbb{C}\right)=: C_{\mathbb{C}}$ the $\mathbb{C}$-vector space of all the complex valued functions which are continuous, by $C\left(\mathbb{Q}_{p}^{n}, \mathbb{R}\right)=: C_{\mathbb{R}}$ the $\mathbb{R}$-vector space of continuous functions. Set

$$
C_{0}\left(\mathbb{Q}_{p}^{n}, \mathbb{C}\right):=C_{0}\left(\mathbb{Q}_{p}^{n}\right)=\left\{f: \mathbb{Q}_{p}^{n} \rightarrow \mathbb{C} ; f \text { is continuous and } \lim _{\|x\|_{p} \rightarrow \infty} f(x)=0\right\},
$$

where $\lim _{\|x\|_{p} \rightarrow \infty} f(x)=0$ means that for every $\epsilon>0$ there exists a compact subset $B(\epsilon)$ such that $|f(x)|<\epsilon$ for $x \in \mathbb{Q}_{p}^{n} \backslash B(\epsilon)$. We recall that $\left(C_{0}\left(\mathbb{Q}_{p}^{n}, \mathbb{C}\right),\|\cdot\|_{L^{\infty}}\right)$ is a Banach space.

2.3. Fourier transform. Set $\chi_{p}(y)=\exp \left(2 \pi i\{y\}_{p}\right)$ for $y \in \mathbb{Q}_{p}$. The map $\chi_{p}(\cdot)$ is an additive character on $\mathbb{Q}_{p}$, i.e. a continuous map from $\left(\mathbb{Q}_{p},+\right)$ into $S$ (the unit circle considered as multiplicative group) satisfying $\chi_{p}\left(x_{0}+x_{1}\right)=\chi_{p}\left(x_{0}\right) \chi_{p}\left(x_{1}\right)$, $x_{0}, x_{1} \in \mathbb{Q}_{p}$. The additive characters of $\mathbb{Q}_{p}$ form an Abelian group which is isomorphic to $\left(\mathbb{Q}_{p},+\right)$, the isomorphism is given by $\xi \mapsto \chi_{p}(\xi x)$, see e.g. [2, Section 2.3]. Given $x=\left(x_{1}, \ldots, x_{n}\right), \xi=\left(\xi_{1}, \ldots, \xi_{n}\right) \in \mathbb{Q}_{p}^{n}$, we set $x \cdot \xi:=\sum_{j=1}^{n} x_{j} \xi_{j}$. If $f \in L^{1}\left(\mathbb{Q}_{p}^{n}\right)$, its Fourier transform is defined by

$$
(\mathcal{F} f)(\xi)=\mathcal{F}_{x \rightarrow \xi}(f)=\widehat{f}(\xi):=\int_{\mathbb{Q}_{p}^{n}} \chi_{p}(\xi \cdot x) f(x) d^{n} x, \quad \text { for } \xi \in \mathbb{Q}_{p}^{n} .
$$

The inverse Fourier transform of a function $f \in L^{1}\left(\mathbb{Q}_{p}^{n}\right)$ is

$$
\left(\mathcal{F}^{-1} f\right)(x)=\mathcal{F}_{\xi \rightarrow x}^{-1}(f)=\int_{\mathbb{Q}_{p}^{n}} \chi_{p}(-x \cdot \xi) f(\xi) d^{n} \xi, \quad \text { for } x \in \mathbb{Q}_{p}^{n} .
$$

The Fourier transform is a linear isomorphism from $\mathcal{D}\left(\mathbb{Q}_{p}^{n}\right)$ onto itself satisfying

$$
(\mathcal{F}(\mathcal{F} f))(\xi)=f(-\xi)
$$

for every $f \in \mathcal{D}\left(\mathbb{Q}_{p}^{n}\right)$, see e.g. [2, Section 4.8].

The Fourier transform $\mathcal{F}(f)=\mathcal{F}_{x \rightarrow \xi}(f)=\widehat{f}$ of a distribution $f$ is defined by the relation

$$
\langle\mathcal{F}(f), \varphi\rangle=\langle f, \mathcal{F}(\varphi)\rangle, \text { for all } \varphi \in \mathcal{D}\left(\mathbb{Q}_{p}^{n}\right) .
$$

The Fourier transform $f \rightarrow \mathcal{F}(f)$ is a linear isomorphism from $\mathcal{D}^{\prime}\left(\mathbb{Q}_{p}^{n}\right)$ onto $\mathcal{D}^{\prime}\left(\mathbb{Q}_{p}^{n}\right)$, see e.g. [2, Section 4.9].

\section{Generalized Bessel potentials}

The goal of this section is to introduce a large class of non-archimedean pseudodifferential operators on $\mathcal{D}\left(\mathbb{Q}_{p}^{n}\right)$ which we call generalized Bessel potentials. 
Definition 1. A function $\psi: \mathbb{Q}_{p}^{n} \rightarrow \mathbb{C}$ is called negative definite, if

$$
\sum_{i, j=1}^{m}\left(\psi\left(x_{i}\right)+\overline{\psi\left(x_{j}\right)}-\psi\left(x_{i}-x_{j}\right)\right) \lambda_{i} \overline{\lambda_{j}} \geq 0
$$

for all $m \in \mathbb{N} \backslash\{0\}, x_{1}, \ldots, x_{m} \in \mathbb{Q}_{p}^{n}, \lambda_{1}, \ldots, \lambda_{m} \in \mathbb{C}$.

Example 1. (i) [17, Example 3.5] For $\alpha$ and $\beta$ positive real numbers, we have that $\boldsymbol{\psi}\left(\|\xi\|_{p}\right)=\alpha\|\xi\|_{p}^{\beta}$ is a negative definite function, where $\|\cdot\|_{p}$ denotes the $p$-adic norm on $\mathbb{Q}_{p}^{n}$. Note that $\boldsymbol{\psi}$ is increasing function with respect to $\|\cdot\|_{p}$.

(ii) Let $f(\xi) \in \mathbb{Q}_{p}^{n}\left[\xi_{1}, \ldots, \xi_{n}\right]$ be a non-constant polynomial. We say that $f(\xi)$ is an elliptic polynomial of degree $d$, if it satisfies: $(a) f(\xi)$ is a homogeneous polynomial of degree $d$, and $(b) f(\xi)=0 \Leftrightarrow \xi=0$. For any $\beta>0$ we have that $|f(\xi)|_{p}^{\beta}$ is a negative definite function, where $|\cdot|_{p}$ denotes the $p$-adic norm on $\mathbb{Q}_{p}$, see [8, Theorem 3]. On the other hand, by [21, Lemma 25], there exist positive constants $C_{0}=C_{0}(f), C_{1}=C_{1}(f)$ such that

$$
C_{0}\|\xi\|_{p}^{d} \leq|f(\xi)|_{p} \leq C_{1}\|\xi\|_{p}^{d}, \text { for every } \xi \in \mathbb{Q}_{p}^{n} .
$$

(iii) [17, Lemma 3.8] Set $\psi_{0}(\xi):=\sum_{j=1}^{\infty} c_{j}\|\xi\|_{p}^{\alpha_{j}}$ with $c_{j} \geq 0, \alpha_{j} \in \mathbb{N}$ such that the real series $\sum_{j=1}^{\infty} c_{j} y^{\alpha_{j}}$ defines a non-constant real function. Then for any $j \in \mathbb{N} \backslash\{0\}$,

$$
\boldsymbol{\psi}\left(\|\xi\|_{p}\right):=e^{e^{. \cdot e^{\psi_{0}(\xi)}}}, j-\text { powers }
$$

is a continuous and negative definite function on $\mathbb{Q}_{p}^{n}$. In this case there is a fixed positive constant $\beta:=\beta(\psi)$ such that for any $\xi \in \mathbb{Q}_{p}^{n}$ we have that $\boldsymbol{\psi}\left(\|\xi\|_{p}\right)>\|\xi\|_{p}^{\beta}$. Moreover, note that $\boldsymbol{\psi}$ is increasing function with respect to $\|\cdot\|_{p}$.

(iv) [18, Remark 3-(ii)] We set $\mathbb{R}_{+}:=\{x \in \mathbb{R}: x \geq 0\}$. Let $J: \mathbb{Q}_{p}^{n} \rightarrow$ $\mathbb{R}_{+}$be a radial (i.e. $J(x)=J\left(\|x\|_{p}\right)$ ) and continuous function such that $\int_{\mathbb{Q}_{p}^{n}} J\left(\|x\|_{p}\right) d^{n} x=1$. Then, the function $\widehat{J}(0)-\widehat{J}\left(\|\xi\|_{p}\right)=1-\widehat{J}\left(\|\xi\|_{p}\right)$ is negative definite. Moreover, $0 \leq 1-\widehat{J}\left(\|\xi\|_{p}\right) \leq 2$ for all $\xi \in \mathbb{Q}_{p}^{n}$.

(v) [4, Proposition 7.4-(iii)] The non-negative constant functions are negative definite.

Lemma 1. Let $f: \mathbb{Q}_{p}^{n} \rightarrow \mathbb{C}$ be a radial function, i.e. $f(x)=f\left(\|x\|_{p}\right)$ for all $x \in \mathbb{Q}_{p}^{n}$. Then $f$ is a locally constant function.

Proof. By [2, Sections 1.8 and 1.10] we have that

$$
\mathbb{Q}_{p}^{n}=\bigsqcup_{\gamma \in \mathbb{Z}} S_{\gamma}^{n}
$$

Let $x \in \mathbb{Q}_{p}^{n}$ fixed. Then, there exists a unique $\gamma:=\gamma(x) \in \mathbb{Z}$ such that $x \in S_{\gamma}^{n}$. Since $f$ is a constant function on $S_{\gamma}^{n}$, then by [2, Proposition 1.8.8 and Section 1.10] and 2, Theorem 1.8.1-(1)] we obtain the results desired.

Remark 1. (i) If $f: \mathbb{Q}_{p}^{n} \rightarrow \mathbb{C}$ is a radial function, then, as a consequence of the previous lemma and [19, Chapter VI-Section 1] we have that $f$ is a continuous function on $\mathbb{Q}_{p}^{n}$.

(ii) If $f: \mathbb{Q}_{p}^{n} \rightarrow \mathbb{C}$ is a radial function, then, it is clear that $|f|$ is a locally constant function on $\mathbb{Q}_{p}^{n}$. 
Definition 2. [Hypothesis A] Let $\boldsymbol{\psi}_{1}, \boldsymbol{\psi}_{2}: \mathbb{Q}_{p}^{n} \rightarrow \mathbb{C}$ be functions. We say that $\boldsymbol{\psi}_{1}$ and $\boldsymbol{\psi}_{2}$ satisfies the Hypothesis $A$ if the following properties are met:

(i) $\boldsymbol{\psi}_{1}$ and $\boldsymbol{\psi}_{2}$ are negative definite and radial (consequently, continuous) functions with $\boldsymbol{\psi}_{1}\left(\|\xi\|_{p}\right) \neq 0$ for all $\xi \in \mathbb{Q}_{p}^{n}$.

(ii) There is a ball $B_{r}^{n}, r:=r\left(\boldsymbol{\psi}_{1}, \boldsymbol{\psi}_{2}\right) \in \mathbb{Z}$, such that

$$
\left|\boldsymbol{\psi}_{1}\left(\|\xi\|_{p}\right)\right| \geq\left|\boldsymbol{\psi}_{2}\left(\|\xi\|_{p}\right)\right| \text { if and only if } \xi \in B_{r}^{n} .
$$

The condition (ii) in the Definition 2 is motivated by [19, Chapter 1-Section I-3]. Denote by $\mathbb{N}:=\{1,2, \ldots\}$ the set of natural numbers and let $\mathbb{R}_{+}:=\{x \in \mathbb{R}: x \geq 0\}$. Throughout this paper we will assume that $\boldsymbol{\psi}_{1}$ and $\boldsymbol{\psi}_{2}$ are functions satisfying the Hypothesis A.

Definition 3. If $f \in \mathcal{D}^{\prime}\left(\mathbb{Q}_{p}^{n}\right), \alpha \in \mathbb{C}$, we define the non-archimedean generalized Bessel potential of order $\alpha$ of $f$ by

$$
\mathcal{F}\left(\mathcal{A}^{\alpha} f\right)=\left[\max \left\{\left|\boldsymbol{\psi}_{1}\left(\|\xi\|_{p}\right)\right|,\left|\boldsymbol{\psi}_{2}\left(\|\xi\|_{p}\right)\right|\right\}\right]^{-\alpha} \widehat{f} .
$$

Let $\alpha \in \mathbb{C}$ fixed. For $\varphi \in \mathcal{D}\left(\mathbb{Q}_{p}^{n}\right)$ we define

$$
\begin{aligned}
\left(\mathcal{A}^{\alpha} \varphi\right)(x) & =\mathcal{F}_{\xi \rightarrow x}^{-1}\left(\left[\max \left\{\left|\boldsymbol{\psi}_{1}\left(\|\xi\|_{p}\right)\right|,\left|\boldsymbol{\psi}_{2}\left(\|\xi\|_{p}\right)\right|\right\}\right]^{-\alpha} \widehat{\varphi}(\xi)\right) \\
& =\int_{\mathbb{Q}_{p}^{n}} \chi_{p}(-x \cdot \xi)\left[\max \left\{\left|\boldsymbol{\psi}_{1}\left(\|\xi\|_{p}\right)\right|,\left|\boldsymbol{\psi}_{2}\left(\|\xi \mid\|_{p}\right)\right|\right\}\right]^{-\alpha} \widehat{\varphi}(\xi) d^{n} \xi, x \in \mathbb{Q}_{p}^{n} .
\end{aligned}
$$

Lemma 2. The application

$$
\begin{aligned}
\mathcal{A}^{\alpha}: \mathcal{D}\left(\mathbb{Q}_{p}^{n}\right) & \rightarrow \mathcal{D}\left(\mathbb{Q}_{p}^{n}\right) \\
\alpha & \longrightarrow \mathcal{A}^{\alpha} \varphi
\end{aligned}
$$

corresponds to a well-defined p-adic pseudo-differential operator where your symbol $\left[\max \left\{\left|\boldsymbol{\psi}_{1}\left(\|\xi\|_{p}\right)\right|,\left|\boldsymbol{\psi}_{2}\left(\|\xi\|_{p}\right)\right|\right\}\right]^{-\alpha} \in \varepsilon\left(\mathbb{Q}_{p}^{n}\right)$.

Proof. Note that

$$
\left[\max \left\{\left|\boldsymbol{\psi}_{1}\left(\|\xi\|_{p}\right)\right|,\left|\boldsymbol{\psi}_{2}\left(\|\xi\|_{p}\right)\right|\right\}\right]^{-\alpha}=\left|\boldsymbol{\psi}_{1}\left(\|\xi\|_{p}\right)\right|^{-\alpha} 1_{B_{r}^{n}}+\left|\boldsymbol{\psi}_{2}\left(\|\xi\|_{p}\right)\right|^{-\alpha} 1_{\mathbb{Q}_{p}^{n} \backslash B_{r}^{n}} .
$$

Given the fact that $1_{B_{r}^{n}}$ and $1_{\mathbb{Q}_{p}^{n} \backslash B_{r}^{n}}$ assume the values 0 and 1 only, and in addition, as the set $\varepsilon\left(\mathbb{Q}_{p}^{n}\right)$ is linear over the field $\mathbb{C}$, see e.g. [19, Chapter VI-Section 1], then by Lemma 1, Remark 1. $(i i)$ and [19, Example 5-p. 80] we have that the function $\left[\max \left\{\left|\boldsymbol{\psi}_{1}\left(\|\xi\|_{p}\right)\right|,\left|\boldsymbol{\psi}_{2}\left(\| \xi||_{p}\right)\right|\right\}\right]^{-\alpha}$ is locally constant on $\mathbb{Q}_{p}^{n}$.

Therefore, if $\varphi \in \mathcal{D}\left(\mathbb{Q}_{p}^{n}\right)$ then by [19, Theorem 4.8.2] we have that $\mathcal{A}^{\alpha} \varphi \in \mathcal{D}\left(\mathbb{Q}_{p}^{n}\right)$.

The function $\mathcal{A}^{\alpha} \varphi, \alpha \in \mathbb{C}$, is called the generalized Bessel potential of order $\alpha$ of $\varphi$.

\section{Convolution kernels attached to generalized Bessel potentials AND ITS APPLICATIONS}

In this section we study certain properties and applications corresponding to the convolution kernels attached to generalized Bessel potentials. From now on, $\alpha$ is a real number such that $\alpha \in \mathbb{R}_{+} \backslash\{0\}$.

We begin with the following definition. 
Definition 4. We define the convolution kernel $K_{\alpha}$ of the generalized Bessel potential $\mathcal{A}^{\alpha}$ by

$$
K_{\alpha}(x):=\int_{\mathbb{Q}_{p}^{n}} \chi_{p}(-x \cdot \xi)\left[\max \left\{\left|\boldsymbol{\psi}_{1}\left(|| \xi \mid \|_{p}\right)\right|,\left|\boldsymbol{\psi}_{2}\left(|| \xi \mid \|_{p}\right)\right|\right\}\right]^{-\alpha} d^{n} \xi, x \in \mathbb{Q}_{p}^{n} .
$$

Remark 2. Note that the symbol $\left[\max \left\{\left|\boldsymbol{\psi}_{1}\left(\|\xi\|_{p}\right)\right|,\left|\boldsymbol{\psi}_{2}\left(\|\xi\|_{p}\right)\right|\right\}\right]^{-\alpha}$ of the pseudodifferential operator $\mathcal{A}^{\alpha}$ defines a regular distribution on $\mathbb{Q}_{p}^{n}$. In this case, by 2 , Proposition 4.9.1] we have that $K_{\alpha} \in \mathcal{D}^{\prime}\left(\mathbb{Q}_{p}^{n}\right)$.

Theorem 1. The convolution kernel $K_{\alpha}$ satisfies the following conditions:

(i) $K_{\alpha} * \varphi=\mathcal{A}^{\alpha} \varphi$, for all $\varphi \in \mathcal{D}\left(\mathbb{Q}_{p}^{n}\right)$.

(ii) For $x \in \mathbb{Q}_{p}^{n} \backslash\{0\}$, we have that

$$
\begin{aligned}
K_{\alpha}(x)= & \|x\|_{p}^{-n}\left\{( 1 - p ^ { - n } ) \sum _ { j = 0 } ^ { \infty } \left(\left[\max \left\{\left|\boldsymbol{\psi}_{1}\left(\|x\|_{p}^{-1} p^{-j}\right)\right|,\left|\boldsymbol{\psi}_{2}\left(\|x\|_{p}^{-1} p^{-j}\right)\right|\right\}\right]^{-\alpha}\right.\right. \\
& \left.\left.-\left[\max \left\{\left|\boldsymbol{\psi}_{1}\left(\|x\|_{p}^{-1} p\right)\right|,\left|\boldsymbol{\psi}_{2}\left(\|x\|_{p}^{-1} p\right)\right|\right\}\right]^{-\alpha}\right) p^{-n j}\right\} .
\end{aligned}
$$

Proof. (i) By using Fubini's Theorem, for $\varphi \in \mathcal{D}\left(\mathbb{Q}_{p}^{n}\right)$ and $x \in \mathbb{Q}_{p}^{n}$ we have that

$$
\begin{aligned}
\left(\mathcal{A}^{\alpha} \varphi\right)(x) & =\int_{\mathbb{Q}_{p}^{n}} \int_{\mathbb{Q}_{p}^{n}} \chi_{p}(y-x \cdot \xi)\left[\max \left\{\left|\boldsymbol{\psi}_{1}\left(|| \xi \mid \|_{p}\right)\right|,\left|\boldsymbol{\psi}_{2}\left(\|\xi\|_{p}\right)\right|\right\}\right]^{-\alpha} \varphi(y) d^{n} y d^{n} \xi \\
& =\int_{\mathbb{Q}_{p}^{n}} \int_{\mathbb{Q}_{p}^{n}} \overline{\chi_{p}(x-y \cdot \xi)}\left[\max \left\{\left|\boldsymbol{\psi}_{1}\left(|| \xi \mid \|_{p}\right)\right|,\left|\boldsymbol{\psi}_{2}\left(\|\xi\|_{p}\right)\right|\right\}\right]^{-\alpha} \varphi(y) d^{n} y d^{n} \xi \\
& =\int_{\mathbb{Q}_{p}^{n}} \int_{\mathbb{Q}_{p}^{n}} \overline{\chi_{p}(z \cdot \xi)}\left[\max \left\{\left|\boldsymbol{\psi}_{1}\left(\|\xi\|_{p}\right)\right|,\left|\boldsymbol{\psi}_{2}\left(\|\xi \mid\|_{p}\right)\right|\right\}\right]^{-\alpha} \varphi(x-z) d^{n} z d^{n} \xi \\
& =\int_{\mathbb{Q}_{p}^{n}} \int_{\mathbb{Q}_{p}^{n}} \chi_{p}(-z \cdot \xi)\left[\max \left\{\left|\boldsymbol{\psi}_{1}\left(\|\xi\|_{p}\right)\right|,\left|\boldsymbol{\psi}_{2}\left(\|\xi\|_{p}\right)\right|\right\}\right]^{-\alpha} d^{n} \xi \varphi(x-z) d^{n} z \\
& =\int_{\mathbb{Q}_{p}^{n}} K_{\alpha}(z) \varphi(x-z) d^{n} z \\
& =\left(K_{\alpha} * \varphi\right)(x) .
\end{aligned}
$$

(ii) Let $x=p^{\gamma} x_{0} \neq 0$ with $\gamma \in \mathbb{Z}$ and $\left\|x_{0}\right\|_{p}=1$. Using the changes of variables $w=p^{\gamma} \xi$ and $z=p^{j} w$, respectively, we have that

$$
\begin{aligned}
K_{\alpha}(x) & =\int_{\mathbb{Q}_{p}^{n}} \chi_{p}\left(-p^{\gamma} \xi \cdot x_{0}\right)\left[\max \left\{\left|\boldsymbol{\psi}_{1}\left(\|\xi\|_{p}\right)\right|,\left|\boldsymbol{\psi}_{2}\left(\|\xi\|_{p}\right)\right|\right\}\right]^{-\alpha} d^{n} \xi \\
& =\|x\|_{p}^{-n} \sum_{-\infty<j<\infty}\left[\max \left\{\left|\boldsymbol{\psi}_{1}\left(p^{\gamma+j}\right)\right|,\left|\boldsymbol{\psi}_{2}\left(p^{\gamma+j}\right)\right|\right\}\right]^{-\alpha} \int_{\|w\|_{p}=p^{j}} \chi_{p}\left(-w \cdot x_{0}\right) d^{n} w \\
& =\|x\|_{p}^{-n} \sum_{-\infty<j<\infty}\left[\max \left\{\left|\boldsymbol{\psi}_{1}\left(p^{\gamma+j}\right)\right|,\left|\boldsymbol{\psi}_{2}\left(p^{\gamma+j}\right)\right|\right\}\right]_{\| p^{j}}^{-\alpha} \int_{w \|_{p}=1} \chi_{p}\left(-w \cdot x_{0}\right) d^{n} w \\
& =\|x\|_{p}^{-n} \sum_{-\infty<j<\infty}\left[\max \left\{\left|\boldsymbol{\psi}_{1}\left(p^{\gamma+j}\right)\right|,\left|\boldsymbol{\psi}_{2}\left(p^{\gamma+j}\right)\right|\right\}\right]_{\|z\| \|_{p}=1}^{-\alpha} \chi_{p}\left(-p^{-j} x_{0} \cdot z\right) d^{n} z
\end{aligned}
$$


By using the formula

$$
\int_{\|z\|_{p}=1} \chi_{p}\left(-p^{-j} x_{0} \cdot z\right) d^{n} z=\left\{\begin{array}{lll}
1-p^{-n}, & \text { if } & j \leq 0 \\
-p^{-n}, & \text { if } j=1 \\
0, & \text { if } j \geq 2,
\end{array}\right.
$$

we have that

$$
\begin{aligned}
K_{\alpha}(x)= & \|x\|_{p}^{-n}\left\{\left(1-p^{-n}\right) \sum_{j=0}^{\infty} p^{-n j}\left[\max \left\{\left|\boldsymbol{\psi}_{1}\left(\|x\|_{p}^{-1} p^{-j}\right)\right|,\left|\boldsymbol{\psi}_{2}\left(\|x\|_{p}^{-1} p^{-j}\right)\right|\right\}\right]^{-\alpha}\right. \\
& \left.-\left[\max \left\{\left|\boldsymbol{\psi}_{1}\left(\|x\|_{p}^{-1} p\right)\right|,\left|\boldsymbol{\psi}_{2}\left(\|x\|_{p}^{-1} p\right)\right|\right\}\right]^{-\alpha}\right\} .
\end{aligned}
$$

Since $\left(1-p^{-n}\right) \sum_{j=0}^{\infty} p^{-n j}=1$, then by (4.3) the desired is obtained.

As a direct consequence of Theorem 1 - $(i i)$ and the fact that $K_{\alpha}(0)>0$, we have the following Corollary.

Corollary 1. If the function $\left[\max \left\{\left|\boldsymbol{\psi}_{1}\left(\|x\|_{p}\right)\right|,\left|\boldsymbol{\psi}_{2}\left(\|x\|_{p}^{-1}\right)\right|\right\}\right]^{-\alpha}$ is increasing with respect to $\|\cdot\|_{p}$, then $K_{\alpha}(x) \geq 0$ for all $x \in \mathbb{Q}_{p}^{n}$.

Example 2. Let $\boldsymbol{\psi}_{1}$ and $\boldsymbol{\psi}_{2}$ be functions given by $\boldsymbol{\psi}_{1}(\xi)=1$ and $\boldsymbol{\psi}_{2}(\xi)=\|\xi\|_{p}^{\beta}$, fixed $\beta>\frac{n}{\alpha}$, for all $\xi \in \mathbb{Q}_{p}^{n}$. Then, by Example 1 , we have that the functions $\boldsymbol{\psi}_{1}$ and $\boldsymbol{\psi}_{2}$ are negative definite radial functions on $\mathbb{Q}_{p}^{n}$.

On the other hand, it is easy to check that $\boldsymbol{\psi}_{1}\left(\|\xi\|_{p}\right) \geq \boldsymbol{\psi}_{2}\left(\|\xi\|_{p}\right)$ if and only if $\xi \in \mathbb{Z}_{p}^{n}$.

By defining for $\alpha>0$

$$
K_{\alpha}(x):=\int_{\mathbb{Q}_{p}^{n}} \chi_{p}(-x \cdot \xi)\left[\max \left\{1,\|\xi\|_{p}^{\beta}\right\}\right]^{-\alpha} d^{n} \xi, x \in \mathbb{Q}_{p}^{n},
$$

by a direct calculation one verifies that $K_{\alpha}(x) \geq 0$, for all $x \in \mathbb{Q}_{p}^{n}$.

Next we will obtain some relevant applications corresponding to the convolution kernels $K_{\alpha}, \alpha>0$.

Definition 5. A function $f: \mathbb{Q}_{p}^{n} \rightarrow \mathbb{C}$ is called positive definite, if

$$
\sum_{i, j=1}^{m} f\left(x_{i}-x_{j}\right) \lambda_{i} \overline{\lambda_{j}} \geq 0
$$

for all $m \in \mathbb{N}, x_{1}, \ldots, x_{m} \in \mathbb{Q}_{p}^{n}$ and $\lambda_{1}, \ldots, \lambda_{m} \in \mathbb{C}$.

Lemma 3. The function $\left[\max \left\{\left|\boldsymbol{\psi}_{1}\left(\|\xi\|_{p}\right)\right|,\left|\boldsymbol{\psi}_{2}\left(\| \xi||_{p}\right)\right|\right\}\right]^{-\alpha}: \mathbb{Q}_{p}^{n} \rightarrow \mathbb{R}_{+} \backslash\{0\}$ is positive definite.

Proof. We first note that

$$
\begin{aligned}
{\left[\max \left\{\left|\boldsymbol{\psi}_{1}\left(\|x-y\|_{p}\right)\right|,\left|\boldsymbol{\psi}_{2}\left(\|x-y\|_{p}\right)\right|\right\}\right]^{-\alpha} } & =\left[\max \left\{\left|\boldsymbol{\psi}_{1}\left(\|y-x \mid\|_{p}\right)\right|,\left|\boldsymbol{\psi}_{2}\left(\|y-x\|_{p}\right)\right|\right\}\right]^{-\alpha} \\
& =\left[\max \left\{\left|\boldsymbol{\psi}_{2}\left(\| y-x||_{p}\right)\right|,\left|\boldsymbol{\psi}_{1}\left(\|y-x\|_{p}\right)\right|\right\}\right]^{-\alpha}
\end{aligned}
$$

for all $x, y \in \mathbb{Q}_{p}^{n}$.

Let $c_{1}, c_{2}, \ldots, c_{m}$ be integer numbers and $\xi_{1}, \xi_{2}, \ldots, \xi_{m} \in \mathbb{Q}_{p}^{n}, m \in \mathbb{N}$. Then, by a 
direct calculation one verifies that

$$
\sum_{i, j=1}^{m}\left[\max \left\{\left|\boldsymbol{\psi}_{1}\left(|| \xi_{i}-\xi_{j} \|_{p}\right)\right|,\left|\boldsymbol{\psi}_{2}\left(\left\|\xi_{i}-\xi_{j} \mid\right\|_{p}\right)\right|\right\}\right]^{-\alpha} c_{i} c_{j}
$$

is exactly $\sum_{i, j=1}^{m}\left[\max \left\{\left|\boldsymbol{\psi}_{1}\left(\left\|\xi_{i}-\xi_{j}\right\|_{p}\right)\right|,\left|\boldsymbol{\psi}_{2}\left(\left\|\xi_{i}-\xi_{j}\right\|_{p}\right)\right|\right\}\right]^{-\alpha} c_{i}^{2} \geq 0$.

On the other hand, it is clear that

$$
\left[\max \left\{\left|\boldsymbol{\psi}_{1}\left(\|-x\|_{p}\right)\right|,\left|\boldsymbol{\psi}_{2}\left(\|-x\|_{p}\right)\right|\right\}\right]^{-\alpha}=\left[\max \left\{\left|\boldsymbol{\psi}_{1}\left(\|x\|_{p}\right)\right|,\left|\boldsymbol{\psi}_{2}\left(\|x\| \|_{p}\right)\right|\right\}\right]^{-\alpha},
$$

for all $x \in \mathbb{Q}_{p}^{n}$.

Therefore, the desired result follow from [4, Exercise 3.7-p. 13].

Lemma 4. $K_{\alpha}$ is a positive bounded measure on $\mathbb{Q}_{p}^{n}$.

Proof. By [2, Proposition 4.9.1], Remark 2 and (4.1) we have that

$$
\mathcal{F}_{x \rightarrow \xi}\left(K_{\alpha}\right)=\left[\max \left\{\left|\boldsymbol{\psi}_{1}\left(\|x\|_{p}\right)\right|,\left|\boldsymbol{\psi}_{2}\left(\|x\|_{p}\right)\right|\right\}\right]^{-\alpha} \in \mathcal{D}^{\prime}\left(\mathbb{Q}_{p}^{n}\right) .
$$

The result follow from Lemma 3, [2, Section 4.9] and [4, Theorem 3.12].

Theorem 2. Suppose that $\left|\boldsymbol{\psi}_{1}(0)\right| \geq 1$. Then, the family $\left(K_{\alpha}\right)_{\alpha>0}$ determine a convolution semigroup on $\mathbb{Q}_{p}^{n}$, i.e. $\left(K_{\alpha}\right)_{\alpha>0}$ satisfies the following properties:

(i) For all $\alpha>0, K_{\alpha}$ is a positive bounded measure on $\mathbb{Q}_{p}^{n}$.

(ii) For all $\alpha>0, K_{\alpha}\left(\mathbb{Q}_{p}^{n}\right) \leq 1$.

(iii) For all $\alpha_{1}, \alpha_{2}>0$ we have that $K_{\alpha_{1}} * K_{\alpha_{2}}=K_{\alpha_{1}+\alpha_{2}}$.

(iv) $\lim _{\alpha \rightarrow 0} K_{\alpha}=\delta$, where $\delta$ is the Dirac delta function.

Proof.

(i) The result follows from Lemma 4 .

(ii) By Lemma 4 and [4, (7)-p. 14] we have that $\left(K_{\alpha}\right)\left(\mathbb{Q}_{p}^{n}\right)=\frac{1}{\left|\psi_{1}(0)\right|^{\alpha}}$. Therefore, if $\left|\boldsymbol{\psi}_{1}(0)\right| \geq 1$, then $\left(K_{\alpha}\right)\left(\mathbb{Q}_{p}^{n}\right) \leq 1$.

(iii) Let $\alpha_{1}$ and $\alpha_{2}$ be real numbers such that $\alpha_{1}, \alpha_{2}>0$. Then, by [2, Proposition 4.9.1], Remark 2. Lemma 2 and (4.1) we have for $i=1,2, \mathcal{F}_{x \rightarrow \xi}\left(K_{\alpha_{i}}\right)=$ $\left[\max \left\{\left|\boldsymbol{\psi}_{1}\left(\|x\|_{p}\right)\right|,\left|\boldsymbol{\psi}_{2}\left(\|x\|_{p}\right)\right|\right\}\right]^{-\alpha_{i}} \in \varepsilon\left(\mathbb{Q}_{p}^{n}\right) \cap \mathcal{D}^{\prime}\left(\mathbb{Q}_{p}^{n}\right)$. Moreover, by [2, Theorem 4.9.3] and [2, Lemma 4.7.2], respectively, there exist integers $N_{i}:=N_{i}\left(\alpha_{i}\right)$, such that $\operatorname{supp}\left(K_{\alpha_{i}}\right) \subset B_{N_{i}}^{n}, i=1,2$, and the convolution $K \alpha_{1} * K \alpha_{2}$ exists.

On the other hand, by the $n$-dimensional version of [19, Theorem-p. 115] and [19, Examples 1 and 2- p. 113 y 114, respectively], we have that the product $\left[\max \left\{\left|\boldsymbol{\psi}_{1}\right|,\left|\boldsymbol{\psi}_{2}\right|\right\}\right]^{-\alpha_{1}}\left[\max \left\{\left|\boldsymbol{\psi}_{1}\right|,\left|\boldsymbol{\psi}_{2}\right|\right\}\right]^{-\alpha_{2}}$ exists and

$$
\mathcal{F}_{\xi \rightarrow x}^{-1}\left(\left[\max \left\{\left|\boldsymbol{\psi}_{1}\right|,\left|\boldsymbol{\psi}_{2}\right|\right\}\right]^{-\alpha_{1}}\left[\max \left\{\left|\boldsymbol{\psi}_{1}\right|,\left|\boldsymbol{\psi}_{2}\right|\right\}\right]^{-\alpha_{2}}\right)=K_{\alpha_{1}} * K_{\alpha_{2}}
$$

Therefore, for $\varphi \in \mathcal{D}\left(\mathbb{Q}_{p}^{n}\right)$ we have that

$$
\begin{aligned}
\left\langle K_{\alpha_{1}+\alpha_{2}}, \varphi\right\rangle & =\left\langle\mathcal{F}_{\xi \rightarrow x}^{-1}\left(\left[\max \left\{\left|\boldsymbol{\psi}_{1}\right|,\left|\boldsymbol{\psi}_{2}\right|\right\}\right]^{-\left(\alpha_{1}+\alpha_{2}\right)}\right), \varphi\right\rangle \\
& =\left\langle\mathcal{F}_{\xi \rightarrow x}^{-1}\left(\left[\max \left\{\left|\boldsymbol{\psi}_{1}\right|,\left|\boldsymbol{\psi}_{2}\right|\right\}\right]^{-\alpha_{1}}\left[\max \left\{\left|\boldsymbol{\psi}_{1}\right|,\left|\boldsymbol{\psi}_{2}\right|\right\}\right]^{-\alpha_{2}}\right), \varphi\right\rangle \\
& =\left\langle K_{\alpha_{1}} * K_{\alpha_{2}}, \varphi\right\rangle .
\end{aligned}
$$

(iv) The result follows from Remark 2 and [19, Example 9-p. 44 and Chapter 1Section VI].

As a direct consequence of above theorem, we have the following corollary.

Corollary 2. If $\left|\boldsymbol{\psi}_{1}(0)\right|=1$, then $K_{\alpha}, \alpha>0$, is a probability measure on $\mathbb{Q}_{p}^{n}$. 


\section{The Green function and the heat Kernel}

In this section we will study certain properties corresponding to the Green function and the heat Kernel attached to operator $\mathcal{A}^{\alpha}$.

5.1. The Green function. The Dirac $\delta$-function is defined by

$$
\langle\delta, \varphi\rangle=\varphi(0), \forall \varphi \in \mathcal{D}\left(\mathbb{Q}_{p}^{n}\right) .
$$

It is clear that $\delta \in \mathcal{D}^{\prime}\left(\mathbb{Q}_{p}^{n}\right)$ and $\delta(x)=0$ for all $x \neq 0$, i.e. $\operatorname{supp}(\delta)=\{0\}$, see [2], 19] for details.

Definition 6. Let $G:=G(m, \alpha) \in \mathcal{D}^{\prime}\left(\mathbb{Q}_{p}^{n}\right)$ be a distribution satisfying the equation

$$
\left(m^{2}+\mathcal{A}^{\alpha}\right) G=\delta, \quad m \in \mathbb{R}_{+} \backslash\{0\} .
$$

Then $G$ is called a Green function of the operator $\mathcal{A}^{\alpha}$.

Lemma 5. The distribution

$$
G(x):=\mathcal{F}_{\xi \rightarrow x}^{-1}\left(\frac{1}{m^{2}+\left[\max \left\{\left|\boldsymbol{\psi}_{1}\left(\|\xi\|_{p}\right)\right|,\left|\boldsymbol{\psi}_{2}\left(\| \xi||_{p}\right)\right|\right\}\right]^{-\alpha}}\right), x \in \mathbb{Q}_{p}^{n},
$$

is the Green function of the operator $\mathcal{A}^{\alpha}$.

Proof. Since $G \in L_{l o c}^{1}\left(\mathbb{Q}_{p}^{n}\right)$, then $G$ determine a regular distribution by the formula

$$
\langle G, \varphi\rangle=\int_{\mathbb{Q}_{p}^{n}} G(x) \varphi(x) d^{n} x, \varphi \in \mathcal{D}\left(\mathbb{Q}_{p}^{n}\right) .
$$

Then, for any $\varphi \in \mathcal{D}\left(\mathbb{Q}_{p}^{n}\right)$ we have that

$$
\begin{aligned}
& \left\langle\left(m^{2}+\mathcal{A}^{\alpha}\right) G, \varphi\right\rangle \\
& =\left\langle G,\left(m^{2}+\mathcal{A}^{\alpha}\right) \varphi\right\rangle \\
& =\left\langle\mathcal{F}_{\xi \rightarrow x}^{-1}\left(\frac{1}{m^{2}+\left[\max \left\{\left|\boldsymbol{\psi}_{1}\left(\|\xi\|_{p}\right)\right|,\left|\boldsymbol{\psi}_{2}\left(\|\xi\|_{p}\right)\right|\right\}\right]^{-\alpha}}\right),\left(m^{2}+\mathcal{A}^{\alpha}\right) \varphi\right\rangle \\
& =\left\langle\frac{1}{m^{2}+\left[\max \left\{\left|\boldsymbol{\psi}_{1}\left(\|\xi\|_{p}\right)\right|,\left|\boldsymbol{\psi}_{2}\left(\|\xi\|_{p}\right)\right|\right\}\right]^{-\alpha}},\left(\left[\max \left\{\left|\boldsymbol{\psi}_{1}\left(\|\xi\|_{p}\right)\right|,\left|\boldsymbol{\psi}_{2}\left(\|\xi \mid\|_{p}\right)\right|\right\}\right]^{-\alpha}+m^{2}\right) \hat{\varphi}\right\rangle \\
& =<1, \widehat{\varphi}> \\
& =<\delta, \varphi>
\end{aligned}
$$

All the above shows that

$$
\left(m^{2}+\mathcal{A}^{\alpha}\right) G=\delta, \quad m \in \mathbb{R}_{+} \backslash\{0\} .
$$

Therefore, $G$ is the Green function of the operator $\mathcal{A}^{\alpha}$.

Theorem 3. The Green function $G$ satisfies the following properties:

(i) For all $x \in \mathbb{Q}_{p}^{n} \backslash\{0\}$ we have that

$$
\begin{aligned}
G(x) & =\|x\|_{p}^{-n}\left\{\left(1-p^{-n}\right) \sum_{j=0}^{\infty} \frac{p^{-n j}}{m^{2}+\left[\max \left\{\left|\boldsymbol{\psi}_{1}\left(p^{\gamma-j}\right)\right|,\left|\boldsymbol{\psi}_{2}\left(p^{\gamma-j}\right)\right|\right\}\right]^{-\alpha}}\right. \\
- & \left.\frac{1}{m^{2}+\left[\max \left\{\left|\boldsymbol{\psi}_{1}\left(p^{\gamma+1}\right)\right|,\left|\boldsymbol{\psi}_{2}\left(p^{\gamma+1}\right)\right|\right\}\right]^{-\alpha}}\right\} .
\end{aligned}
$$


(ii) If $\left[\max \left\{\left|\boldsymbol{\psi}_{1}\left(\|\xi\|_{p}\right)\right|,\left|\boldsymbol{\psi}_{2}\left(\|\xi\|_{p}\right)\right|\right\}\right] \geq 1$ for all $\xi \in \mathbb{Q}_{p}^{n}$, then, there exist positive real constants $K_{1}:=\frac{1}{m^{2}\left(m^{2}+1\right)}$ and $K_{2}:=\frac{1}{m^{2}}$ such that

$$
-K_{1}\|x\|_{p}^{-n} \leq G(x) \leq K_{2}\|x\|_{p}^{-n}, \text { for all } x \in \mathbb{Q}_{p}^{n} \backslash\{0\},
$$

(iii) $G(x)$ is a real-valued, radial and continuous function.

Proof. (i) Let $x=p^{\gamma} x_{0} \neq 0$ with $\gamma \in \mathbb{Z}$ and $\left\|x_{0}\right\|_{p}=1$. Then,

$$
G(x)=\int_{\mathbb{Q}_{p}^{n}} \chi_{p}\left(-p^{\gamma} \xi \cdot x_{0}\right)\left(\frac{1}{m^{2}+\left[\max \left\{\left|\boldsymbol{\psi}_{1}\left(\|\xi\|_{p}\right)\right|,\left|\boldsymbol{\psi}_{2}\left(\|\xi\|_{p}\right)\right|\right\}\right]^{-\alpha}}\right) d^{n} \xi .
$$

We make a change of variables, namely $z=p^{\gamma} \xi$, we have that $G(x)$ is exactly

$$
\begin{aligned}
& \|x\|_{p}^{-n} \sum_{-\infty<j<\infty}\left(\frac{1}{m^{2}+\left[\max \left\{\left|\boldsymbol{\psi}_{1}\left(p^{\gamma}|| z \mid \|_{p}\right)\right|,\left|\boldsymbol{\psi}_{2}\left(p^{\gamma}|| z \mid \|_{p}\right)\right|\right\}\right]^{-\alpha}}\right) \int_{\|z\|_{p}=p^{j}} \chi_{p}\left(-z \cdot x_{0}\right) d^{n} z \\
& =\|x\|_{p}^{-n} \sum_{-\infty<j<\infty}\left(\frac{1}{m^{2}+\left[\max \left\{\left|\boldsymbol{\psi}_{1}\left(p^{\gamma+j}\right)\right|,\left|\boldsymbol{\psi}_{2}\left(p^{\gamma+j}\right)\right|\right\}\right]^{-\alpha}}\right) \int_{\left\|p^{j} z\right\|_{p}=1} \chi_{p}\left(-z \cdot x_{0}\right) d^{n} z \\
& =\|x\|_{p}^{-n} \sum_{-\infty<j<\infty}\left(\frac{p^{n j}}{m^{2}+\left[\max \left\{\left|\boldsymbol{\psi}_{1}\left(p^{\gamma+j}\right)\right|,\left|\boldsymbol{\psi}_{2}\left(p^{\gamma+j}\right)\right|\right\}\right]^{-\alpha}}\right) \int_{\|z\|_{p}=1} \chi_{p}\left(-p^{-j} x_{0} \cdot z\right) d^{n} z
\end{aligned}
$$

By using the formula (4.2), we obtain the desired equality.

(ii) By $(i)$ and taking $K_{2}:=\frac{1}{m^{2}}$ we have that

$$
\begin{aligned}
G(x) & \leq\|x\|_{p}^{-n}\left\{\left(1-p^{-n}\right) \sum_{j=0}^{\infty}\left(\frac{p^{-n j}}{m^{2}+\left[\max \left\{\left|\boldsymbol{\psi}_{1}\left(p^{\gamma-j}\right)\right|,\left|\boldsymbol{\psi}_{2}\left(p^{\gamma-j}\right)\right|\right\}\right]^{-\alpha}}\right)\right\} \\
& \leq\|x\|_{p}^{-n}\left\{\frac{1}{m^{2}} \sum_{j=0}^{\infty}\left(p^{-n j}-p^{-n(j+1)}\right)\right\} \\
& =K_{2}\|x\|_{p}^{-n} .
\end{aligned}
$$

On the other hand, by (i) and taking $K_{1}:=\frac{1}{m^{2}\left(m^{2}+1\right)}$ we have that

$$
\begin{aligned}
G(x) & \geq\|x\|_{p}^{-n}\left\{\left(1-p^{-n}\right) \sum_{j=0}^{\infty}\left(\frac{p^{-n j}}{m^{2}+1}\right)-\frac{1}{m^{2}+\left[\max \left\{\left|\boldsymbol{\psi}_{1}\left(p^{\gamma+1}\right)\right|,\left|\boldsymbol{\psi}_{2}\left(p^{\gamma+1}\right)\right|\right\}\right]^{-\alpha}}\right\} \\
& \geq\|x\|_{p}^{-n}\left\{\frac{1}{m^{2}+1} \sum_{j=0}^{\infty}\left(p^{-n j}-p^{-n(j+1)}\right)-\frac{1}{m^{2}}\right\} \\
& =-K_{1}\|x\|_{p}^{-n} .
\end{aligned}
$$

(iii) Is a direct consequence of (i), Lemma 5 and Remark 1- $(i)$. 
5.2. The heat kernel. We assume throughout this section that $\left|\boldsymbol{\psi}_{1}\right|$ and $\left|\boldsymbol{\psi}_{2}\right|$ are increasing functions with respect to $\|\cdot\|_{p}$.

We define the heat Kernel (also called fundamental solution) attached to operator $\mathcal{A}^{\alpha}$ as

$$
Z_{t}(x)=Z(x, t):=\int_{\mathbb{Q}_{p}^{n}} \chi_{p}(-x \cdot \xi) e^{-t\left[\max \left\{\left|\boldsymbol{\psi}_{1}\left(\|x\| \|_{p}\right)\right|,\left|\boldsymbol{\psi}_{2}\left(\|x\| \|_{p}\right)\right|\right\}\right]^{-\alpha}} d^{n} \xi,
$$

for $x \in \mathbb{Q}_{p}^{n}$ and $t \geq 0$.

Remark 3. If $t=0$, then by [2, Example 4.9.1] we have that

$$
Z_{0}(x)=\int_{\mathbb{Q}_{p}^{n}} \chi_{p}(-x \cdot \xi) d^{n} \xi=\delta \in \mathcal{D}^{\prime}\left(\mathbb{Q}_{p}^{n}\right) .
$$

For $t>0$ and by a direct calculation one verifies that $e^{-t\left[\max \left\{\left|\boldsymbol{\psi}_{1}\right|,\left|\boldsymbol{\psi}_{2}\right|\right\}\right]^{-\alpha}} \notin L^{1}\left(\mathbb{Q}_{p}^{n}\right)$. Now, by using the fact that $e^{-t\left[\max \left\{\left|\boldsymbol{\psi}_{1}\right|,\left|\boldsymbol{\psi}_{2}\right|\right\}\right]^{-\alpha}} \in L_{\text {loc }}^{1}\left(\mathbb{Q}_{p}^{n}\right)$, then $e^{-t\left[\max \left\{\left|\boldsymbol{\psi}_{1}\right|,\left|\boldsymbol{\psi}_{2}\right|\right\}\right]^{-\alpha}}$ defines a regular distribution. Therefore, by [2, Proposition 4.9.1] we have that $Z_{t}(x) \in \mathcal{D}^{\prime}\left(\mathbb{Q}_{p}^{n}\right)$.

Theorem 4. $Z_{t}(x) \leq 0$ for all $x \in \mathbb{Q}_{p}^{n} \backslash\{0\}$ and $t>0$.

Proof. Let $x=p^{\gamma} x_{0} \neq 0$ such that $\gamma \in \mathbb{Z}$ and $\left\|x_{0}\right\|_{p}=1$. Then, by (5.2) and using the changes of variables $w=p^{\gamma} \xi$ and $z=p^{j} w$, respectively, we have that

$$
\begin{aligned}
Z(x, t) & =\|x\|_{p}^{-n} \int_{\mathbb{Q}_{p}^{n}} \chi_{p}\left(-w \cdot x_{0}\right) e^{-t\left[\max \left\{\left|\boldsymbol{\psi}_{1}\left(|| w \mid \|_{p} p^{\gamma}\right)\right|,\left|\boldsymbol{\psi}_{2}\left(\|w\|_{p} p^{\gamma}\right)\right|\right\}\right]^{-\alpha}} d^{n} w \\
& =\|x\|_{p}^{-n} \sum_{-\infty<j<\infty} e^{-t\left[\max \left\{\left|\boldsymbol{\psi}_{1}\left(p^{\gamma+j}\right)\right|,\left|\boldsymbol{\psi}_{2}\left(p^{\gamma+j}\right)\right|\right\}\right]^{-\alpha}} \int_{\left\|p^{j}\right\|_{p}=1} \chi_{p}\left(-w \cdot x_{0}\right) d^{n} w \\
& =\|x\|_{p}^{-n} \sum_{-\infty<j<\infty} e^{-t\left[\max \left\{\left|\boldsymbol{\psi}_{1}\left(p^{\gamma+j}\right)\right|,\left|\boldsymbol{\psi}_{2}\left(p^{\gamma+j}\right)\right|\right\}\right]^{-\alpha}} p^{n j} \int_{\|z\| \|_{p}=1} \chi_{p}\left(-p^{-j} x_{0} \cdot z\right) d^{n} z .
\end{aligned}
$$

By using the formula (4.2) one verifies that

$$
\begin{aligned}
Z(x, t)= & \|x\|_{p}^{-n}\left\{\left(1-p^{-n}\right) \sum_{j=0}^{\infty} e^{-t\left[\max \left\{\left|\boldsymbol{\psi}_{1}\left(\|x\|_{p}^{-1} p^{-j}\right)\right|,\left|\boldsymbol{\psi}_{2}\left(\|x\|_{p}^{-1} p^{-j}\right)\right|\right\}\right]^{-\alpha}} p^{-n j}\right. \\
& \left.-e^{-t\left[\max \left\{\left|\boldsymbol{\psi}_{1}\left(\|x\|_{p}^{-1} p\right)\right|,\left|\boldsymbol{\psi}_{2}\left(\|x\|_{p}^{-1} p\right)\right|\right\}\right]^{-\alpha}}\right\} .
\end{aligned}
$$

Now, we need consider three cases for $\|x\|_{p}$.

Case 1. $\|x\|_{p}>p^{-r}$. In this case, we have that $\|x\|_{p}^{-1}<p^{r}$. Therefore, $\|x\|_{p}^{-1} p^{-j} \leq$

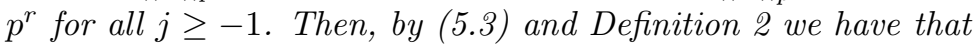

$$
\begin{aligned}
Z(x, t) & =\|x\|_{p}^{-n}\left\{\left(1-p^{-n}\right) \sum_{j=0}^{\infty} e^{-t\left|\boldsymbol{\psi}_{1}\left(\|x\|_{p}^{-1} p^{-j}\right)\right|^{-\alpha}} p^{-n j}-e^{-t\left|\boldsymbol{\psi}_{1}\left(\|x\|_{p}^{-1} p\right)\right|^{-\alpha}}\right\} \\
& \leq\|x\|_{p}^{-n}\left\{e^{-t\left|\boldsymbol{\psi}_{1}\left(\|x\|_{p}^{-1}\right)\right|^{-\alpha}}-e^{-t\left|\boldsymbol{\psi}_{1}\left(\|x\|_{p}^{-1} p\right)\right|^{-\alpha}}\right\} \\
& \leq 0 .
\end{aligned}
$$


Case 2. $\|x\|_{p}=p^{-r}$. In this case, we have that $\|x\|_{p}^{-1}=p^{r}$. Therefore, $\|x\|_{p}^{-1} p^{-j} \leq$ $p^{r}$ for all $j \geq 0$ and moreover, $\|x\|_{p}^{-1} p>p^{r}$. Then, by (5.3) and Definition 2 we have that

$$
\begin{aligned}
Z(x, t) & =\|x\|_{p}^{-n}\left\{\left(1-p^{-n}\right) \sum_{j=0}^{\infty} e^{-t\left|\boldsymbol{\psi}_{1}\left(\|x\|_{p}^{-1} p^{-j}\right)\right|^{-\alpha}} p^{-n j}-e^{-t\left|\boldsymbol{\psi}_{2}\left(\|x\|_{p}^{-1} p\right)\right|^{-\alpha}}\right\} \\
& \leq\|x\|_{p}^{-n}\left\{e^{-t\left|\boldsymbol{\psi}_{1}\left(\|x\|_{p}^{-1}\right)\right|^{-\alpha}}-e^{-t\left|\boldsymbol{\psi}_{2}\left(\|x\|_{p}^{-1} p\right)\right|^{-\alpha}}\right\} \\
& \leq 0 .
\end{aligned}
$$

Case 3. $\|x\|_{p}<p^{-r}$. In this case, we have that $\|x\|_{p}^{-1}>p^{r}$. Therefore, proceeding analogously as in the previous cases, we have that

$$
\begin{aligned}
Z(x, t) & \leq\|x\|_{p}^{-n}\left\{e^{-t\left|\boldsymbol{\psi}_{2}\left(\|x\|_{p}^{-1}\right)\right|^{-\alpha}}-e^{-t\left|\boldsymbol{\psi}_{2}\left(\|x\|_{p}^{-1} p\right)\right|^{-\alpha}}\right\} \\
& \leq 0 .
\end{aligned}
$$

Remark 4. Consider the following p-adic heat equation or Cauchy problem given by

$$
\left\{\begin{array}{l}
\frac{\partial u}{\partial t}(x, t)=\mathcal{A}^{\alpha} u(x, t), \quad t \in[0, \infty), \quad x \in \mathbb{Q}_{p}^{n} \\
u(x, 0)=u_{0}(x) \in \mathcal{D}\left(\mathbb{Q}_{p}^{n}\right),
\end{array}\right.
$$

where $\mathcal{A}^{\alpha}$ is the pseudo-differential operator previously defined.

By proceeding as in the proof of [18, Proposition 1], we have that

$$
u(x, t):=\int_{\mathbb{Q}_{p}^{n}} \chi_{p}(-x \cdot \xi) e^{-t\left[\max \left\{\left|\boldsymbol{\psi}_{1}\left(|| \xi \|_{p}\right)\right|,\left|\boldsymbol{\psi}_{2}\left(\|\xi \mid\|_{p}\right)\right|\right\}\right]^{-\alpha}} \widehat{u_{0}}(\xi) d^{n} \xi,
$$

with, $u_{0}(x) \in \mathcal{D}\left(\mathbb{Q}_{p}^{n}\right), x \in \mathbb{Q}_{p}^{n}$ and $t \geq 0$, is the unique (classical) solution of the Cauchy problem (5.4). Moreover, by [18, Lemma 3] we have that $u(x, t)=Z_{t}(x) * u_{0}$. Since the heat equation describes the distribution of heat (or temperature variations) in a region along the course of time, then by Theorem 4 we have that equation (5.4) describes the cooling (or loss of heat) in a given region over time.

\section{REFERENCES}

[1] Aguilar-Arteaga V., Cruz-López M., Estala-Arias S., Non-Archimedean analysis and a wavetype pseudodifferential equation on finite adèles. J. Pseudo-Differ. Oper. Appl. (2020) DOI: 10.1007/s11868-020-00343-1.

[2] Albeverio S., Khrennikov A. Yu., Shelkovich V. M., Theory of $p$-adic distributions: linear and nonlinear models. London Mathematical Society Lecture Note Series, 370. Cambridge University Press, Cambridge, 2010.

[3] Antoniouk A.V., Khrennikov A.Y., Kochubei A.N., Multidimensional nonlinear pseudodifferential evolution equation with p-adic spatial variables. J. Pseudo-Differ. Oper. Appl. (2019) doi:10.1007/s11868-019-00320-3

[4] Berg Christian, Forst Gunnar, Potential theory on locally compact abelian groups. SpringerVerlag, New York-Heidelberg, 1975.

[5] Bikulov A. Kh.,Investigation of the $p$-adic Green function. Theor. Math. Phys. 87, 376-390 (1991).

[6] Casas-Sánchez, O., Chacón-Cortés, L., Galeano-Peñaloza, J., Semi-linear Cauchy problem and Markov process associated with a $p$-adic non-local ultradiffusion operator. J. PseudoDiffer. Oper. Appl. 11, 10851110 (2020). https://doi.org/10.1007/s11868-020-00334-2 
[7] Dragovich B., Khrennikov A. Yu., Kozyrev S. V., Volovich I. V., On p-adic mathematical physics, P-Adic Numbers Ultrametric Anal. Appl. 1 (1) (2009) 117.

[8] Gutiérrez García I., Torresblanca-Badillo A., Strong Markov processes and negative definite functions associated with non-Archimedean elliptic pseudo-differential operators. J. PseudoDiffer. Oper. Appl. (2019), 1-18.

[9] Gutiérrez García I., Torresblanca-Badillo A., Some classes of non-archimedean pseudodifferential operators related to Bessel potentials, J. Pseudo-Differ. Oper. Appl. (2020) DOI: $10.1007 / \mathrm{s} 11868-020-00333-3$

[10] Khrennikov A., Oleschko K., Correa López M., Modeling Fluids Dynamics with Master Equations in Ultrametric Spaces Representing the Treelike Structure of Capillary Networks. Entropy 2016, 18, 249; doi: 10.3390/e18070249

[11] Khrennikov A. Y., Kochubei A. N., p-Adic Analogue of the Porous Medium Equation. J Fourier Anal Appl. 24, 1401-1424 (2018).

[12] Khrennikov A. Yu., Kozyrev S. V., Zúñiga-Galindo W. A., Ultrametric pseudodifferential equations and applications. Encyclopedia of Mathematics and its applications, Cambridge University Press, 2018. DOI 10.1017/9781316986707

[13] Kochubei A. N., Pseudo-differential equations and stochastic over non-Archimedean fields, Pure and Applied Mathematics 244, Marcel Dekker, New York, MR 2003b:35220 Zbl 0984.11063, 2001

[14] Oleschko K., Khrennikov A. Yu., "Applications of p-adics to geophysics: Linear and quasilinear diffusion of water-in-oil and oil-in-water emulsions", TMF, 190:1 (2017), 179190; Theoret. and Math. Phys., 190:1 (2017), 154163

[15] Pourhadi E., Khrennikov A., Saadati R., Oleschko K., Correa López M., Solvability of the p-adic analogue of Navier-Stokes equation via the wavelet theory. Entropy 2019, 21, 1129; doi: $10.3390 / \mathrm{e} 21111129$

[16] Taibleson M. H., Fourier analysis on local fields. Princeton University Press, 1975.

[17] Torresblanca-Badillo A., Zúñiga-Galindo W. A., Non-Archimedean Pseudodifferential Operators and Feller Semigroups, p-Adic Numbers, Ultrametric Analysis and Applications, Vol. 10, No. 1, pp. 57-73, 2018.

[18] Torresblanca-Badillo A., Zúñiga-Galindo W. A., Ultrametric Diffusion, exponential landscapes, and the first passage time problem, Acta Appl Math (2018), 157:93.

[19] Vladimirov V. S., Volovich I. V., Zelenov E. I., p-adic analysis and mathematical physics. World Scientific, 1994.

[20] Vladimirov V. S., Volovich I. V., p-Adic quantum mechanics. Commun. Math. Phys., 123, 659-676 (1989).

[21] Zúñiga-Galindo W. A., Pseudodifferential Equations Over Non-Archimedean Spaces. Lecture Notes in Mathematics 2174, Springer International Publishing, 2016.

E-mail address: atorresblanca@uninorte.edu.co

Universidad del Norte, Departamento de Matemáticas y Estadística, Km. 5 Vía Puerto Colombia. Barranquilla, Colombia. 